\title{
Robotic Intraoperative Tracheobronchial Repair during Minimally Invasive 3-Stage Esophagectomy
}

\author{
Alessandra Marano, M.D., Silvia Palagi, M.D., Luca Pellegrino, M.D., Felice Borghi, M.D. \\ Department of Surgery, General and Oncologic Surgery Unit, Santa Croce e Carle Hospital, Cuneo, Italy
}

\author{
ARTICLE INFO \\ Received May 20, 2020 \\ Revised July 4, 2020 \\ Accepted July 14, 2020 \\ Corresponding author \\ Felice Borghi \\ Tel 39-0171642288 \\ Fax 39-0171642214 \\ E-mail borghi.f@ospedale.cuneo.it \\ ORCID \\ https://orcid.org/0000-0002-2431-2020
}

\begin{abstract}
Tracheobronchial injury (TBI) is an uncommon but potentially fatal event. latrogenic lesions during bronchoscopy, endotracheal intubation, or thoracic surgery are considered the most common causes of TBI. When TBI is detected during surgery, concomitant surgical treatment is recommended. Herein we present a case of successful robotic primary repair of iatrogenic tracheal and left bronchial branch tears during a robot-assisted hybrid 3-stage esophagectomy after neoadjuvant chemoradiotherapy. A robotic approach can facilitate the repair of this injury while reducing both the potential risk of conversion to open surgery and the associated increased risk of postoperative respiratory complications.
\end{abstract}

Keywords: Tracheal injury, Bronchial injury, Robotics, Esophageal neoplasms, Esophagectomy

\section{Case report}

Tracheobronchial injury (TBI) during esophagectomy is a rare complication that occurs in only $0.6 \%$ to $3.9 \%$ of cases but is associated with significant morbidity and mortality [1]. In the literature, a multifactorial origin of these iatrogenic tears has been proposed, including both anatomical and mechanical risk factors [2]. Several anatomical risk factors have been described, most notably tracheal distortion by mediastinal or endobronchial masses, preoperative radiochemotherapy (especially for the treatment of advanced cancer of the mid-esophagus), female sex, and age greater than 65 years. The mechanical risk factors include mainly procedural or instrumentation-related conditions, such as multiple attempts at intubation or intubation with double-lumen tubes. Furthermore, emergency intubation increases the risk of death threefold compared to elective intubation.

According to the size and location of the damage, treatment is either conservative or surgical [3]. When TBI is detected intraoperatively during thoracic surgery, concomitant surgical treatment is recommended [3]. Moreover, when a primary minimally invasive (MI) thoracoscopic approach is used, conversion to open surgery is possible and carries an increased risk of postoperative respiratory complications [4]. In the present report, we describe a case of robotic primary repair of tracheal and left bronchial branch tears during a McKeown esophagectomy performed at a tertiary referral center. This report was composed in accordance with the criteria set out in the Surgical Case Report checklist [5].

A healthy 76-year-old woman with a body mass index of $20.5 \mathrm{~kg} / \mathrm{m}^{2}$ reported dysphagia, substernal chest pain, and weight loss. Endoscopy with endoscopic ultrasound identified an esophageal squamous cell carcinoma (ESCC) of the middle third of the esophagus with a celiac axis and paracardial lymph nodes (LNs) suspicious for metastatic disease. Computed tomography and positron emission tomography confirmed esophageal wall thickening (maximum standardized uptake value [SUVmax], 16.7) and revealed swollen mediastinal LNs (SUVmax, 6.2) but no distant metastases (Fig. 1). Bronchoscopy showed no invasion of the tumor into the tracheobronchial tree, and no other pathological changes were detected. A preoperative diagnosis of cT3N2M0 stage III ESCC was made. Following a multidisciplinary team consultation, a robot-assisted hybrid 3-stage esophagectomy with lymphadenectomy was planned for 8 weeks after the completion of neoadjuvant chemoradiotherapy. 

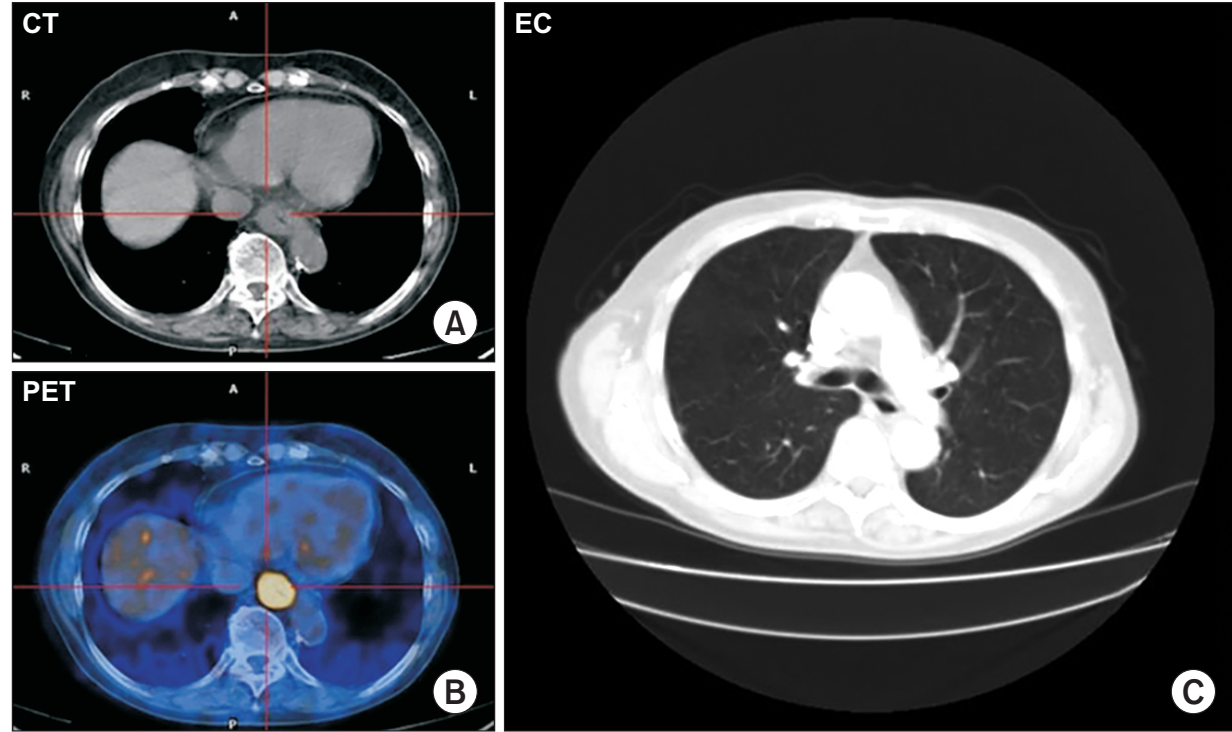

Fig. 1. (A-C) Preoperative images. Computed tomography (CT) revealed esophageal cancer (EC) of the middle third of the esophagus with no evidence of invasion of the tumor into the tracheobronchial tree. Positron emission tomography (PET) confirmed the presence of esophageal wall thickening (maximum standardized uptake value, 16.7).

\section{Operative technique}

To facilitate the thoracic approach, the patient was intubated with a left-sided double-lumen tube and was placed in the left lateral decubitus position, tilted $45^{\circ}$ to the prone position. During patient positioning, repeated endobronchial tube placements were necessary due to tube dislodgement.

Fig. 2 shows the robotic port layout for the chest stage. Starting at the anterior side of the esophagus, the parietal pleura was cut from the level of the azygos arch down to the diaphragm where the pulmonary ligament was divided. The azygos arch was ligated with Weck Hem-o-lok clips (Teleflex, Morrisville, NC, USA). Then, the dissection of the parietal pleura was extended to the esophageal hiatus until the aorta was completely exposed.

Subsequently, surgery was continued above the azygos arch for right paratracheal LN dissection. The right vagus nerve was then sectioned just below the carina, preserving its bronchial branches. The dissection of the esophagus below the tracheal bifurcation was difficult to perform since it was tightly adjoined, and the left endobronchial balloon suddenly became visible. Since no difficulty was being experienced with ventilation, it was decided to complete the dissection before repairing the bronchial tear.

At this stage, a Penrose drain was placed around the esophagus to facilitate traction. The dissection of the esophagus was continued along the pericardium down to the diaphragm. Here, the thoracic duct was clipped with robotic Weck Hem-o-lok clips (Teleflex). The resection of the esophagus en bloc with the surrounding LNs and the

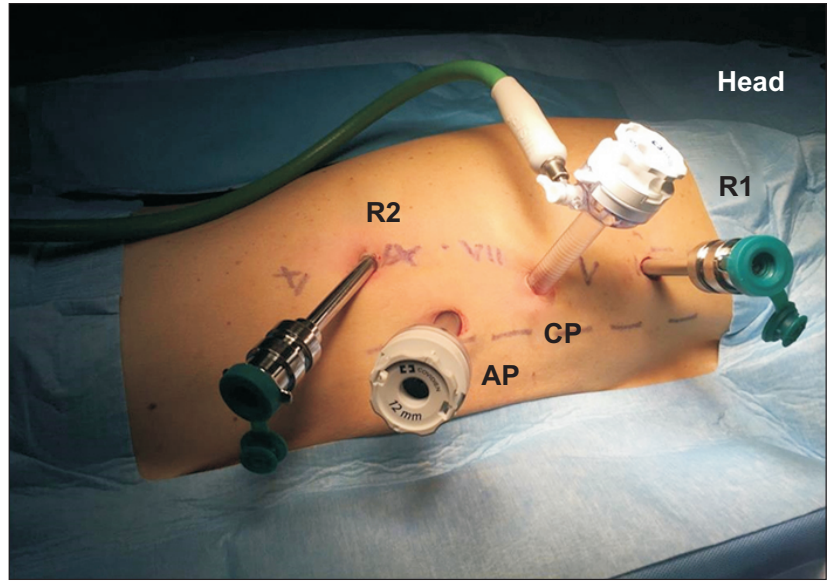

Fig. 2. Robotic thoracoscopic port layout. A 12-mm camera port (CP) for the $30^{\circ}$ down scope was placed at the sixth intercostal space, posterior to the posterior axillary line. Two $8-\mathrm{mm}$ ports were placed anterior to the scapular rim in the fourth intercostal space (R1) and more posterior in the ninth intercostal space (R2). One 12-mm assistant port (AP) was placed in the eighth intercostal space. The da Vinci Si (Intuitive Surgical Inc., Sunnyvale, CA, USA) was docked from the patient's dorsocranial side. Written informed consent for publication of this image was obtained from the family of the patient.

thoracic duct was completed from the diaphragm up to the thoracic inlet. During the posterior dissection at the level of the azygos arch, another defect was detected on the posterior wall of the trachea. Finally, the supra-azygos esophagus and periesophageal tissue were completely resected into the thoracic apex, preserving the recurrent laryngeal nerves. Next, both defects-a $2-\mathrm{cm}$ defect on the posterior aspect of the trachea and a $1.5-\mathrm{cm}$ defect at the level of the 
left main bronchus immediately below the tracheal bifurcation-were repaired with a robot-sewn interrupted $4 / 0$ polydioxanone suture. A final underwater air leak test was negative, and a fibrin sealant patch was applied (Supplementary Video 1).

Following the chest phase, 2 drains were placed, and the lung was insufflated under direct vision. The patient was then placed in the supine position for the open abdominal and cervical stage, and a terminalized semi-mechanical side-to-side cervical esophagogastrostomy was performed.

The total operative time was 480 minutes. The repair of the tracheobronchial lacerations took approximately 60 minutes. The patient was extubated at the end of the procedure with no respiratory disorders. The results of a 10day follow-up bronchoscopy were normal. The patient was discharged on postoperative day 18 without any complications. Final pathology revealed ypT0N0 (0/28 LNs) M0 ESCC. No postoperative tracheobronchial strictures were observed. The patient died 14 months after surgery from mediastinal recurrent disease.

\section{Informed consent}

Written informed consent was obtained from the family of the patient for the publication of this case report, accompanying images, and video.

\section{Discussion}

This case highlights the successful use of a robotic approach for the surgical management of tracheal and left bronchial branch tears during a McKeown esophagectomy for the treatment of ESCC. Multiple risk factors are associated with the occurrence of this uncommon, but potentially fatal complication. In the case presented, repeated double-lumen intubation [2] and sclerotic changes of esophagealtracheal muscle fibers after neoadjuvant chemoradiotherapy for the treatment of advanced middle-third ESCC played a decisive role [1]. Furthermore, the latter condition led to inflammation and local edema, making esophageal dissection and extended mediastinal lymphadenectomy quite difficult to perform [1]. Given this background, the inevitable traction and the effect of thermal cautery during the dissection may have contributed to the extent of both injuries. Lastly, the female sex, advanced age (76 years), and short height $(152 \mathrm{~cm})$ of the patient represented other important risk factors for the injury [2].

Primary surgical repair is highly recommended after an intraoperative diagnosis in cases of a full-layer injury or an injury larger than $2 \mathrm{~cm}$ [3]. These conditions occurred in the case described herein. Multiple options are available regarding the surgical repair procedure and the type of approach, and the appropriate method to use in a given case depends on the characteristics of the lesion [2].

If surgical treatment is planned during primary MI esophagectomy, conversion to open surgery may be needed and can be associated with an increased risk of postoperative respiratory complications. Indeed, in recent decades, the application of MI techniques such as thoracoscopic and robotic approaches for esophageal cancer treatment have proven to be as effective as open surgery in terms of longterm oncological outcomes. Furthermore, these MI approaches carry a reduced rate of cardiopulmonary complications and are associated with a better quality of life [4].

To the best of our knowledge, only 1 case has been reported of intraoperative primary repair of 2 defects of the tracheobronchial tree with thoracoscopic intracorporeal suture during esophageal resection in a patient with ESCC [6]. In the current case, robotic primary repair of tracheal and left-bronchial branch tears during a McKeown esophagectomy proved safe and feasible in experienced hands and in the absence of difficulties with patient ventilation. This system provided excellent exposure and facilitated otherwise complex steps, such as the intracorporeal suture. Moreover, it has the well-known advantages of MI approaches of reduced perioperative morbidity and improved quality of life compared to the open technique.

The following are tips to prevent intraoperative iatrogenic trauma to the trachea and bronchi during MI esophagectomy.

\section{Dedicated team and standardized procedure}

The presence of an experienced team dedicated to MI esophagectomy along with the performance of a standardized procedure may partially mitigate some related risk factors.

\section{Intubation risk}

Some mechanical and instrumentation-related risk factors exist for airway injury during intubation [7]. This risk can further increase during both transhiatal and transthoracic MI esophagectomy when the patient is moved from a supine to a lateral decubitus position after double-lumen tube placement. Therefore, adequate procedure-specific training and education of support staff are crucial. 


\section{Absence of tactile feedback in robotic surgery and the key role of the bedside assistant}

One of the major pitfalls of robotic surgery is the loss of tactile feedback. Thus, clear visualization and exposure are of paramount importance, especially during robotic MI esophagectomy. Hence, the proficiency of the bedside assistant plays a key role.

\section{Type of energy device}

During esophageal mobilization along the pericardium and the dissection of the subcarinal LNs, great care must be taken to avoid energy-associated thermal injury (either direct or due to thermal spread) to the membranous wall of the major airways. The judicious use of ultrasonic devices, bipolar or monopolar energy sources, and blunt dissectors is strongly recommended [8]. The experiences of expert surgeons at other high-volume esophageal centers with robotic MI esophagectomy suggest the use of articulated bipolar energy instrumentation with minimal lateral thermal spread [8].

\section{Conflict of interest}

No potential conflict of interest relevant to this article was reported.

\section{Acknowledgments}

The authors thank Mrs. Anna Maria Racca for her significant contribution to the revision of the manuscript.

\section{ORCID}

Alessandra Marano: https://orcid.org/0000-0002-2904-2170

Silvia Palagi: https://orcid.org/0000-0001-8119-0632

Luca Pellegrino: https://orcid.org/0000-0002-2475-3475
Felice Borghi: https://orcid.org/0000-0002-2431-2020

\section{Supplementary materials}

Supplementary materials can be found via https://doi. org/10.5090/jcs.20.055. Supplementary Video 1. Robotic intraoperative tracheobronchial repair during minimally invasive 3 -stage esophagectomy.

\section{References}

1. Koshenkov VP, Yakoub D, Livingstone AS, Franceschi D. Tracheobronchial injury in the setting of an esophagectomy for cancer: postoperative discovery a bad omen. J Surg Oncol 2014;109:804-7.

2. Grewal HS, Dangayach NS, Ahmad U, Ghosh S, Gildea T, Mehta AC. Treatment of tracheobronchial injuries: a contemporary review. Chest 2019;155:595-604.

3. Lee SK, Kim DH, Lee SK, Kim YD, Cho JS, I H. Does surgical repair still have a role for iatrogenic tracheobronchial rupture?: clinical analysis of a thoracic surgeon's opinion. Ann Thorac Cardiovasc Surg 2016;22:348-53.

4. Van der Sluis PC, van der Horst S, May AM, et al. Robot-assisted minimally invasive thoracolaparoscopic esophagectomy versus open transthoracic esophagectomy for resectable esophageal cancer: a randomized controlled trial. Ann Surg 2019;269:621-30.

5. Agha RA, Borrelli MR, Farwana R, et al. The SCARE 2018 statement: updating consensus Surgical Case Report (SCARE) guidelines. Int J Surg 2018;60:132-6.

6. Khitaryan A, Miziev I, Veliev C, et al. Case report of successful management of intraoperative tracheal rupture during thoracoscopic esophageal resection in patient with esophageal cancer. Int J Surg Case Rep 2019;57:42-6.

7. Minambres E, Buron J, Ballesteros MA, Llorca J, Munoz P, Gonzalez-Castro A. Tracheal rupture after endotracheal intubation: a literature systematic review. Eur J Cardiothorac Surg 2009;35:1056-62.

8. Okusanya OT, Hess NR, Luketich JD, Sarkaria IS. Technique of robotic assisted minimally invasive esophagectomy (RAMIE). J Vis Surg 2017;3:116. 